\title{
The core surveillance system SCORPIO and its validation against measured pressurised-water reactor plant data $\dagger$
}

\author{
ØIVIND BERG, SVERRE HVAL and \\ UFFE SCOT JØRGENSEN‡
}

\begin{abstract}
Keywords: PWR type reactors, in-core instruments, reactor control systems, reactor instrumentation, reactor cores, reactor simulators, computerised control systems, on-line control systems, programming.

The computerised core surveillance system SCORPIO is described. Main modules in the system are an interactive communication system with a function keyboard and a fully graphic colour screen; a strategy generator which proposes how to operate the reactor in the near future; a fast core simulator with predictive capability; a data base and a limit check module which calculates margins to the operational limits. The predictive part of the system is completed, and is already installed at a pressurised-water reactor plant. The core follow module is under development, and installation of this module will take place in 1987. Results from the validation of the strategy generator and the simulator are also described.
\end{abstract}

\section{Introduction}

A growing interest in core surveillance systems has been registered over the last few years. There are several motivating factors which have stimulated the development of these computerised systems. An on-line core simulator-like improved core instrumentation - can make less conservative reactor operation possible. Obviously a detailed core surveillance system has the potential of better fuel utilisation, which leads to economic benefits. However, increased operational safety is also obtained when local margins to limits are monitored on-line, especially in power transients where the xenon redistribution influences the power distribution. A more flexible operation of nuclear power plants also calls for extensive predictive simulations of various control strategies on-site, and can now be realised on fast minicomputers at an acceptable cost.

In the Halden Project, core modeling and development of core control methods have been an ongoing activity for several years. It was decided in 1979 to see how these principles could be utilised on-line for operators in the control room. Development of the SCORPIO (Surveillance of reactor CORe by PIcture On-line display) system was then initiated and the prototype system for predictive analysis was ready in October 1982. This prototype has been adapted to the Swedish Nuclear Power Plant in Ringhals (Units 2, 3 and 4 PWR). At the moment it serves as an aid for the

Received October 11986.

$\dagger$ This paper was printed in Atomkernenergie - Kerntechnik 45 (1984) and is reprinted in MIC with the permission of Carl Hauser Verlag, München, West Germany.

$\ddagger$ OECD Halden Reactor Project, Institutt for Energiteknikk, P.O. Box 173, N-1751 Halden, Norway. 


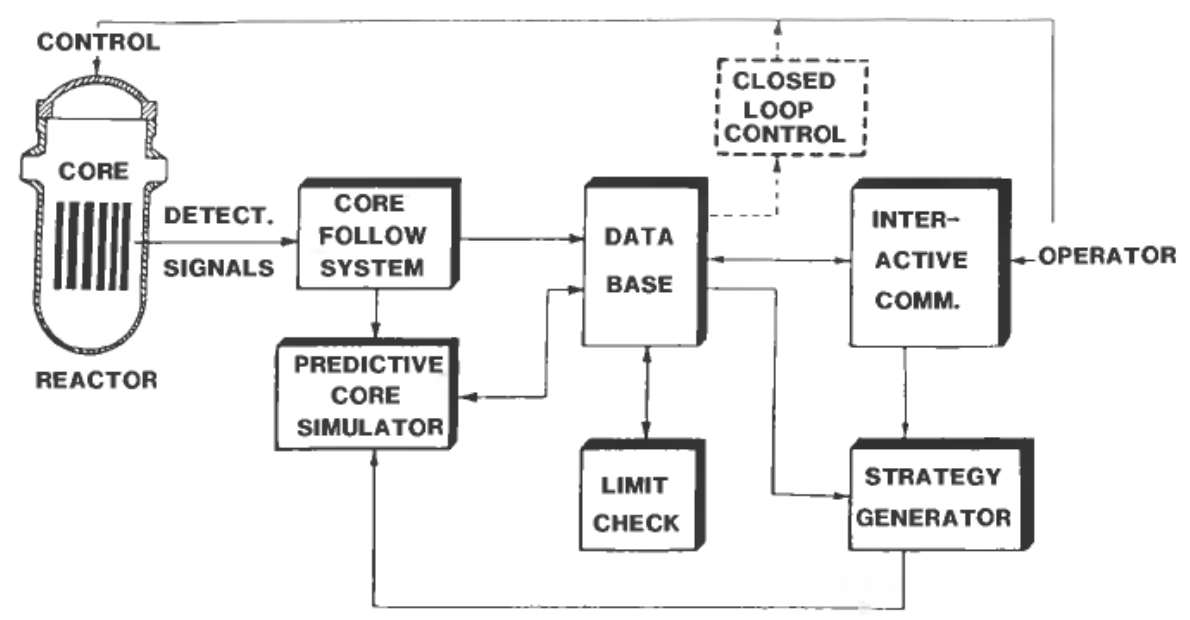

Figure 1. Modules of a complete SCORPIO system.

reactor physicist and a tool for operator training. The intention is to implement the complete system in the control room at a later stage.

Fig. 1 shows the modules of a complete system and their interconnections. The main function of SCORPIO's predictive mode of operation is to assist the operator in deriving good control strategies during power transients. Special weight has therefore been put on making a simple user-friendly operator interface. The operator communicates with the system through a specially designed function keyboard, an alphanumeric keyboard and a fully graphic colour screen. A strategy generator is included to propose controller settings which then can be input to a fast 3dimensional core simulator. A limit check module calculates margins to limits and the results are presented as colour pictures. Speed and flexibility have been obtained by parallel processing techniques and efficient data handling.

Following the reactor core condition as a function of time is another part of the SCORPIO system which is still under development, and a main task in this connection is to combine simulator calculations with measurements to obtain a best possible estimate of the core condition.

\section{Description of physics modules}

\subsection{Strategy generator}

2.1.1. Purpose. The strategy generator module serves two purposes in the SCORPIO system: It helps the user in specifying controller setpoints which keep the power distribution within specified limits during a transient. This increases the efficiency of the system, since a good control strategy is obtained quickly. It also acts as an input generator for the predictive core simulator by proposing average temperature, core flow, boron concentration and rod bank positions during the transient. This makes the system more user-friendly since it eliminates the trivial task of entering in large amounts of data.

2.1.2. The model. In principle, the strategy generator is an extremely simplified core simulator. The power and xenon-iodine densities are calculated for two points, rep- 
resenting the upper and lower core halves, respectively. This is accomplished without solving the neutronic and hydraulic equations which govern the core behaviour. Instead, the strategy generator relies heavily upon precalculated coefficients to predict the reactor's response to changes of power, inlet temperature, control rod positions, boron concentration and xenon concentration. These coefficients are generated by performing a series of simulations of different reactor conditions with the more detailed core simulator. The axial power offset is calculated by assuming that it is a linear function of the total reactor power, the product of relative power and boron concentration and of the difference in Xe-135 concentration between the lower and upper core halves. The following three assumptions are used to account for the influence from control rods:

(1) Rods which are either not inserted or fully inserted do not affect the axial offset.

(2) The maximum influence occurs when the rod is inserted halfway.

(3) For all other positions the rod's influence can be determined by using a linear relation between rod insertion and axial offset, taking the influence from the average xenon concentration on the rod strength into account.

When calculating the effective multiplication factor $k_{\text {eff }}$ of the reactor it is assumed that a linear correlation exists between $k_{\text {eff }}$ and inlet temperature, xenon and boron concentrations and rod bank positions. A quadratic function describes the influence of total reactor power upon $k_{\text {eff }}$. In ideal cases the calculated $k_{\text {eff }}$ should be 1 , since the reactor is supposed to be critical at the starting point of the calculation. However, due to inevitable inaccuracies in the model the calculated $k_{\text {eff }}$ may differ from 1 . By requiring that this deviation stays constant during the transient, the strategy generator is able to calculate the boron concentration and rod bank positions which result in a critical system with satisfactory accuracy. The calculation of the transient behaviour proceeds step by step in time. The xenon and iodine concentrations at the end of a step are calculated by assuming that the power in each half of the core changes linearly during a time step. With this assumption, the equation for the I-135 concentration is solved analytically. A slight modification of the equation for Xe-135 also makes it possible to obtain an analytic solution for the xenon concentration. The new values for the boron concentration and control rod positions which make the reactor critical at the end of a step are found by calculating the deviation of $k_{\text {eff }}$ from the reference value, and then adjusting controllers until this deviation is the same as at the starting point. The physical limitations regarding boron dilution and insertion rates and rod speeds are taken into account. If it is impossible to get the reactor critical at the desired power level, the maximum power for which a critical system is possible is calculated.

The offset is controlled by moving the rods, and an iteration must be performed to adjust both $k_{\text {eff }}$ and the axial offset of the core to the values required. If one does not succeed in attaining good values for both $k_{\text {eff }}$ and the axial offset, the controllers are used so that the reactor becomes critical (with the offset value outside the desired interval).

2.1.3. Validation of the strategy generator. The strategy generator has been compared with the more detailed CYGNUS core simulator for a large number of different transients. 24 transients, each lasting $18 \mathrm{~h}$, have been run for the PWR Grafenrheinfeld (thermal output $3765 \mathrm{MW}$ ) at zero burnup. In most cases the axial 


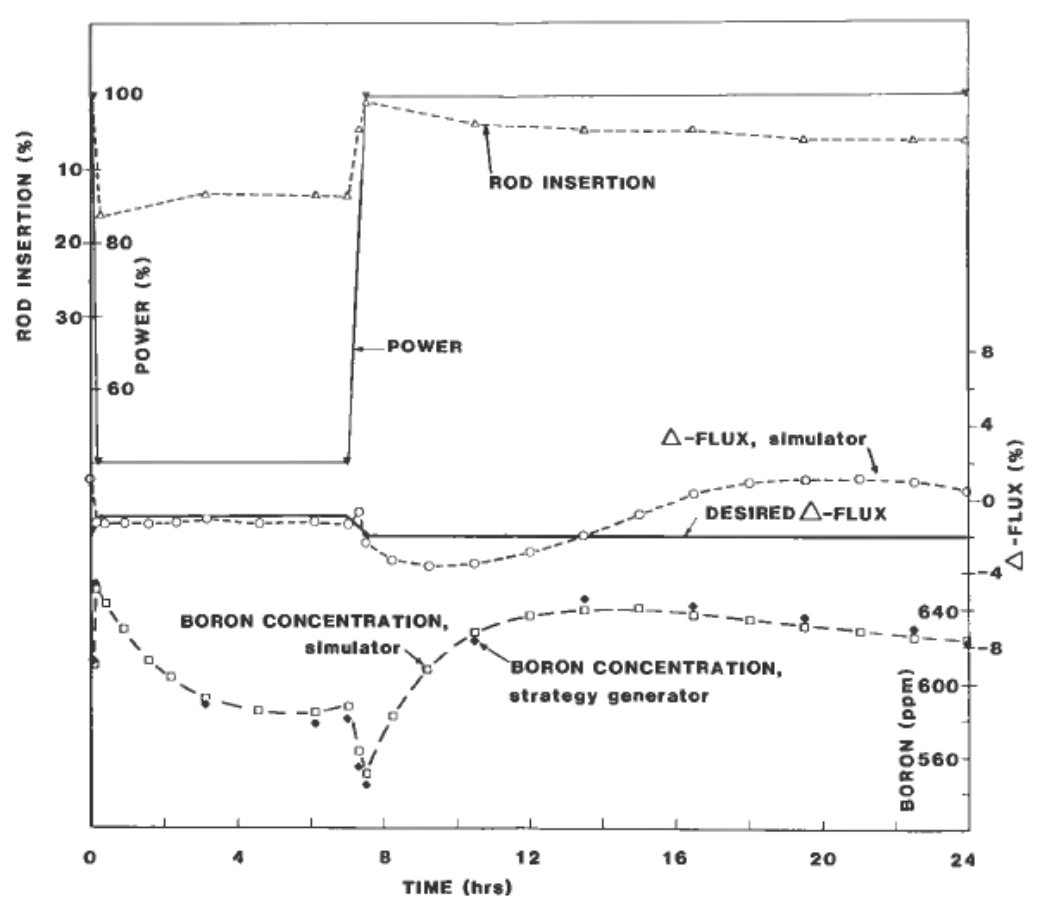

Figure 2. Transient starting with a rapid power reduction from $100 \%$ to $50 \%$.

power offset deviated less than $10 \%$ from the desired value throughout the transient.

Ten transients with a duration of $24 \mathrm{~h}$ have been run for the Westinghouse PWR Ringhals 2 (thermal output $2432 \mathrm{MW}$ ) with data from the beginning of cycle 8 (core average uranium burnup ca. 16,500 $\mathrm{MWd} / \mathrm{t}$ ). The objective in these transients was to keep a quantity called $\Delta$-flux within a band of $\pm 5 \%$ around a power dependent target value ( $\Delta$-flux $=$ axial offset $\times$ relative power). This was achieved with a few exceptions. The results for two of these transients are shown in Figs. 2 and 3.

The transient shown in Fig. 2 is a rapid $(5 \mathrm{~min})$ power reduction from $100 \%$ to $50 \%$. After $7 \mathrm{~h}$ at $50 \%$ power, the reactor power is increased to $100 \%$ in $30 \mathrm{~min}$, and the reactor is then kept at full power. This transient represents a case in which the reactor power must be reduced quickly (for instance, after one of the turbines has tripped) and a fast return to full power is required after the problems have been corrected.

Figure 3 shows the results for a transient in which the power was increased from $50 \%$ equilibrium to $100 \%$. After $10 \mathrm{~h}$ the power was reduced to $20 \%$, where it was kept for some hours before returning to full power. Note that in this case the initial power distribution was considerably more top-peaked than the desired distribution ( $\Delta$-flux value $5 \%$, desired value $-1 \%$ ). 


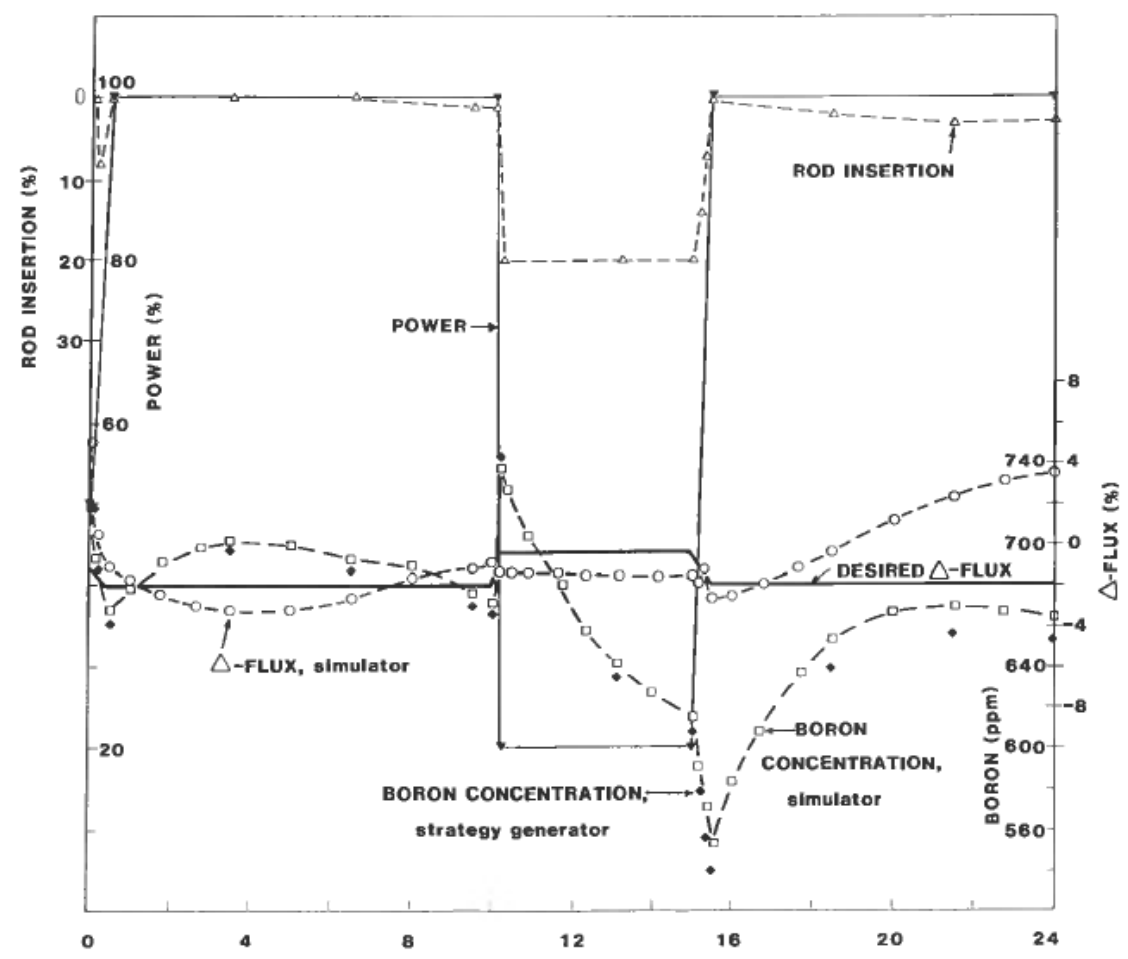

Figure 3. Transient starting with a power increase from $50 \%$ to $100 \%$.

\subsection{Core simulator}

The CYGNUS core simulator used in the core surveillance system is developed especially for this purpose, which means that the following points have been emphasized:

accurate and detailed solution.

fast execution on minicomputers,

ease of use,

programming in standard FORTRAN to facilitate transfer to other machines.

The neutronics part of the simulator employs a coarse mesh nodal method, giving a spatial resolution of about $20 \mathrm{~cm}$. The usual expression for internodal neutron current has been improved by utilising results derived from invariant imbedding theory (Weiss 1972).

While the requirements of computational speed prohibit the use of a full twoenergy group model, standard one-group theory would not give sufficient accuracy. A type of 'one-and-a-half-group' model is introduced, where the thermal flux is eliminated based on the asymptotic flux ratio in the actual node modified by the corresponding ratios in the six neighbouring nodes (Tsuiki et al. 1974, 1976). A further reformulation of the neutron balance equation is made to make it suited for numerical solution. Boundary conditions are treated by geometry dependent flux estimates for the reflector. 


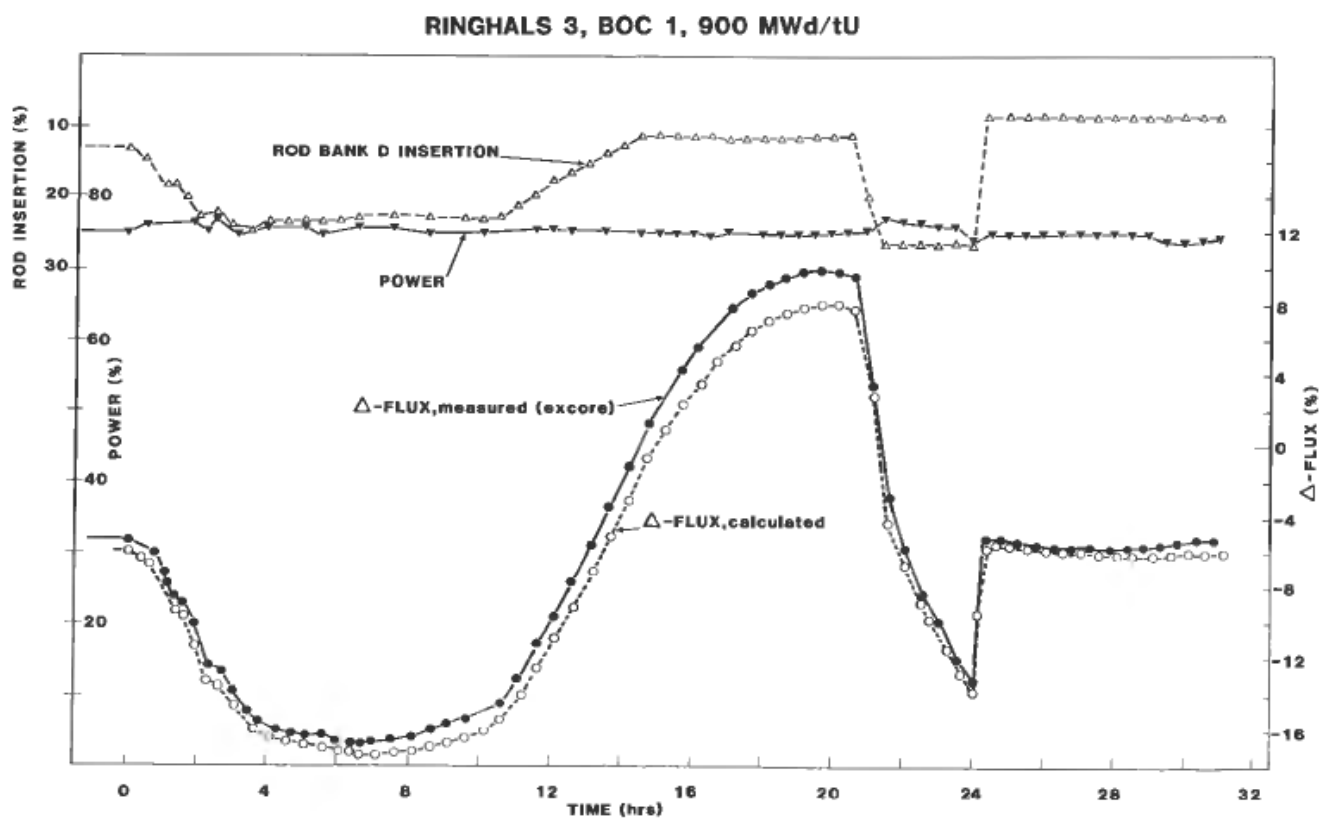

Figure 4. Transient with axial power oscillation induced by control rod motion.

Special care is taken in the formulation of the xenon dynamics equations to include the effect of xenon concentration on the neutron spectrum to enable prediction of the core status several hours into the future. In addition to the thermal absorption cross section, the fission cross section will depend on the xenon concentration. The model also includes changes in the microscopic absorption cross section of Xe-135 as a function of the spectrum. The accuracy of a xenon transient calculation depends, among other factors, on the xenon integration model and the length of the time step. In CYGNUS, the flux is assumed to change as a linear function of time, allowing relatively long time steps. A special feature has been included in the code to simplify the transient specification. It consists of an automatic time step generator which determines the xenon transient time steps allowing for the speed with which certain core variables are changing. The most important parameter influencing the xenon integration step is the rate of change of control rod positions.

It is unlikely that anybody will be able to give input for all controllers in such a way that criticality can be maintained throughout a transient. Therefore the simulator must be able to find the controller positions which will make the reactor critical at every time step. The controllers available for this criticality search in CYGNUS are the boron concentration and the positions of the control rod banks. The sequence in which they are used is determined by user-defined priorities. A check is always performed to ensure that the controller does not exceed maximum or minimum values of insertion (concentration) or rate of change. If this happens, the controller is used as fast as possible, and the next controller is then activated.

The computing time is well within the requirements of an on-line simulator, and on the ND-500 computer (comparable to VAX 11/780) one time step is completed in about $12 \mathrm{~s}$ for a quarter-core PWR calculation with 1120 nodes. For a reasonable transient without too many rapid rod movements, an average step length of about $1 \mathrm{~h}$ is usual, so a predictive calculation covering the next twenty $\mathrm{h}$ requires four min. 
RINGHALS 2, BOC 8, $16500 \mathrm{MWd} / \mathrm{tU}$

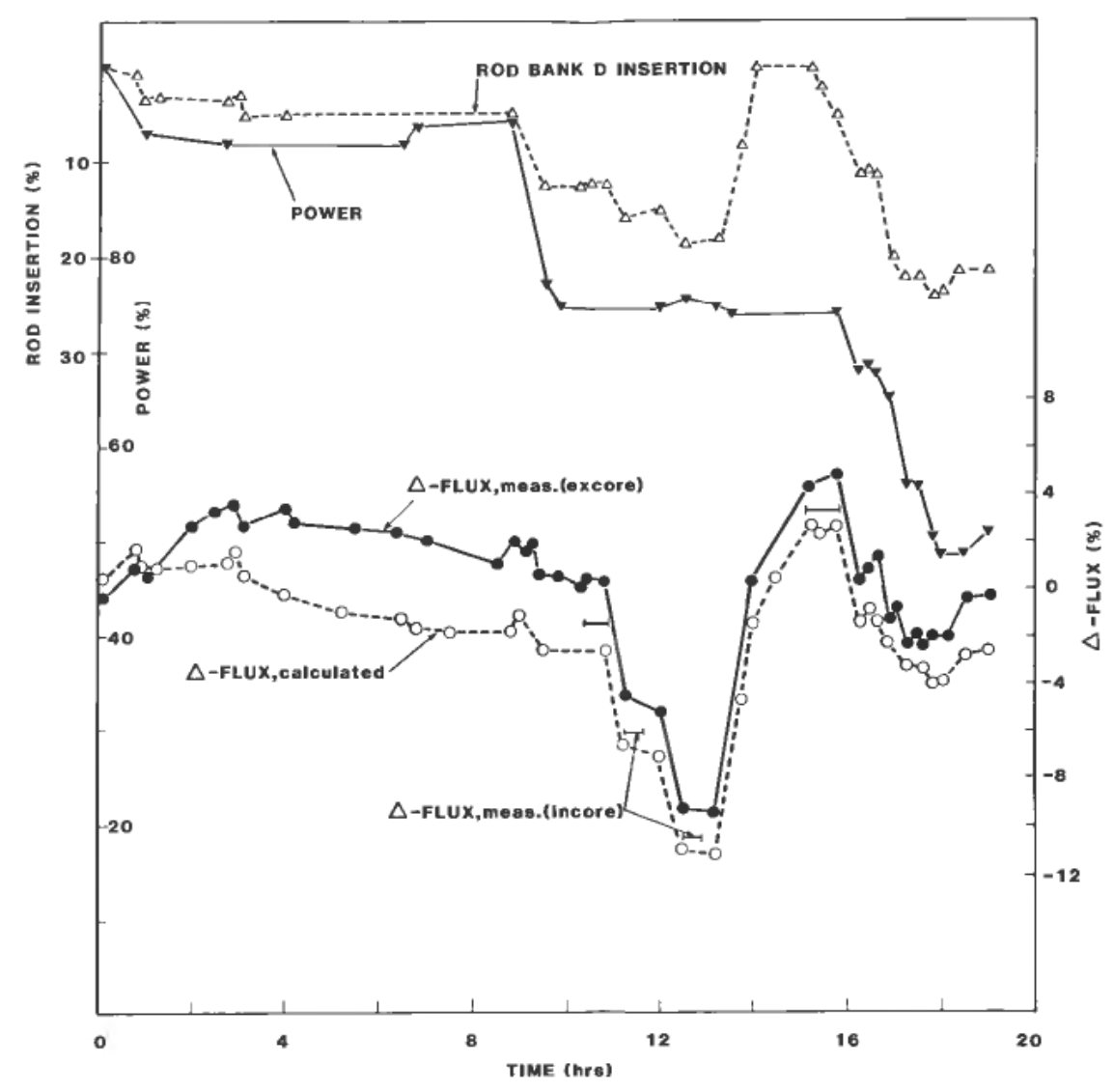

Figure 5. Transient run for the calibration of neutron detectors.

2.2.1. Validation of the simulator. The core simulator has been checked against results from other codes (PDQ-7, QUABOX/CUBBOX). A maximum deviation of $0 \cdot 2 \%$ was observed for $k_{\text {eff }}$, and the maximum error in local power was $7 \%$.

Recently the code has also been checked against measured plant data from the Westinghouse PWRs at Ringhals unit 3, cycle 1, and Ringhals unit 2, cycle 8. Cross section data for these comparisons were taken from CASMO (Ahlin et al. 1977) calculations performed by the Swedish State Power Board. Several xenon transients have been simulated, and the results of this analysis seem to indicate that the simulator is capable of following the process for several days without adjustments and still keep the errors in $\Delta$-flux within a few per cent.

Figure 4 shows results from a $30 \mathrm{~h}$ transient in the $2775 \mathrm{MW}$ (thermal) reactor at unit 3 , which was run at almost constant power $(75 \%)$, and where a strong axial power oscillation was induced by control rod motion. This transient occurred at a uranium burnup of $900 \mathrm{MWd} / \mathrm{t}$ of cycle 1 . During this transient the rms deviation between measured and calculated average channel powers was almost constant at $2 \cdot 5 \%$.

In Fig. 5 the results for a transient which was run to calibrate the excore neutron detectors of unit 2 (2432 MW thermal) in the beginning of cycle 8 (core average uranium burnup ca. $16,500 \mathrm{MWd} / \mathrm{t}$ ) are shown. For this transient measurements 


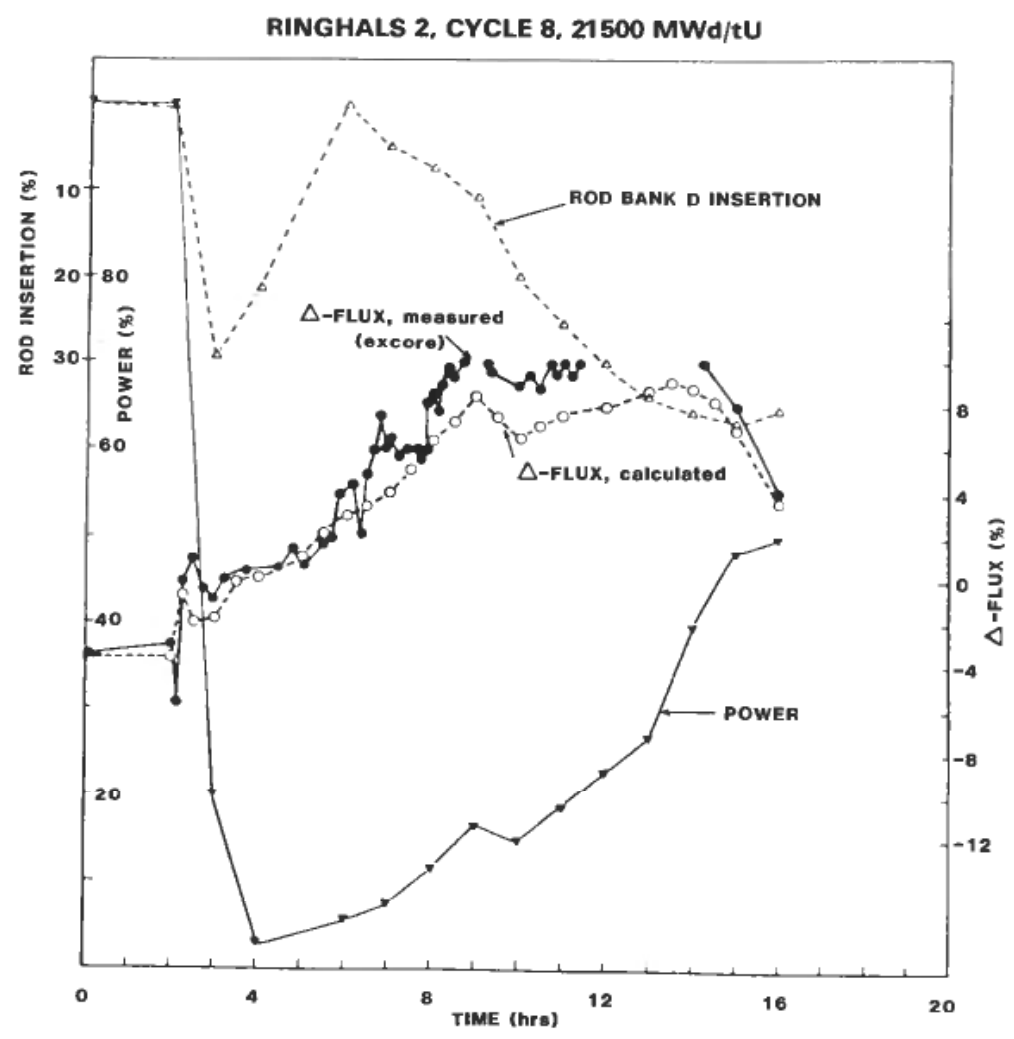

Figure 6. Transient initiated by an unexpected power reduction.

from traversing incore probes (TIP) were also available, and the results from these measurements are shown as horizontal bars in the figure.

Figure 6 shows the comparison between measured and calculated values of $\Delta$-flux during an unexpected power reduction which occurred after $5000 \mathrm{MWd} / \mathrm{t}$ in cycle 8 (core average burnup ca. $21,500 \mathrm{MWd} / \mathrm{t}$ ). Note that the lack of measured data for two periods of this transient is caused by the limited range of the recorder. It should also be noted that the values for power and rod insertions were not recorded as frequently as would have been desirable for a detailed simulation. This transient is an example of a situation in which a predictive core surveillance system would probably be well suited as a tool to assist the operator in avoiding future undesirable operating conditions.

\section{Limit check}

The limit checking module is the module which compares the power distribution and other core conditions against the core operational and safety limitations. At the moment, four different limit checks are included in SCORPIO. These are the two form factors $F_{Q}$ and $F_{\Delta H}, \Delta$-flux and a very simplified PCI (Pellet-Clad Interaction) limitation to prevent fuel failure. The $F_{Q}, F_{\Delta H}$ and $\Delta$-flux limitations are those specified for the Swedish Ringhals 2 reactor. The fuel failure model is very simple, and compares the pin power distribution during a transient with a power envelope 
defined from the equilibrium power distribution. Information on margins to limits is most important to the operator, and several pictures have been developed that give both spatial and trend information on margins. Data sets have therefore been made to produce a detailed map of the axial positions for the most critical node in a channel and a sorted list of the ten most critical nodes in the core at every time step. A data set is prepared to contain the most critical node in the reactor for each of the three limit types. In the case of predictive calculations, an overview trend picture gives minimum margins to all limits during the whole transient. If limits are exceeded, more detailed pictures show where the problem occurs in the core, and a modified predictive run can be defined.

\section{Data base system}

\subsection{Background}

During the design and specification phase of SCORPIO the importance of a clear cut interface between the various modules of the system was stressed. This requirement also influences the way data are stored and transferred in the system. If the same data are used in different modules and at different times, a book-keeping system has to be introduced. Also the speed requirements are quite important when storing data or especially when requesting data for display. No extra time should be spent seeking data on large data files. Finally, maintenance aspects such as deleting old data and storing new data are much improved when a data base system is available.

\subsection{Data set structure}

A basic requirement for SCORPIO is the fast response when a picture is requested. This implies that the division of data into data sets must be picture oriented. One data set, which is the smallest unit transferred between the mass storage files of the data base and the fast memory, or a few data sets should contain all the dynamic information for one picture. Next, one single value is retrieved per process variable from the data sets in the fast memory. Hence the design of the data set layout and the picture layout are strongly related, and this must be taken into account because it determines the response time for the display of results to a large extent. In this way a retrieval time of only a few tenths of a second per data set has been obtained.

\section{Picture design and implementation}

\subsection{Picture summary}

The communication system is designed in order to facilitate preparation of simulator input, to follow and survey the execution of the simulation, and to present core simulation results in a suitable manner for the operator.

For the present SCORPIO version a total of 15 pictures have been designed. These pictures can be divided into simulator input and simulator output. The four simulator input pictures show the variation in time of power, boron concentration, average temperature and a selected control bank's position both graphically and as a table. The power input must be given manually, the other input pictures are to be built up either manually or by using the strategy generator. 
Ten pictures can be characterised as simulator output pictures. They can again be divided in two subgroups. The first contains three pictures showing the time variation (trend) of power and related variables like $\Delta$-flux and margins to different limits $\left(F_{Q}, F_{\Delta H}, P C I\right)$, of boron concentration and average temperature, and of positions for selected control banks.

The second subgroup contains seven pictures for closer inspection of the core. They show spatial distributions of power and related variables, and the status of boron concentration and all control bank positions at a certain selected time.

The layout of these seven pictures is closely related to the nodewise calculation of the power distribution. In addition, there is a picture called strategy overview which identifies the strategies which have been calculated and stored in the data base. The time required to display a picture on the screen is about 2 to $3 \mathrm{~s}$ from when the appropriate button on the function keyboard is pressed.

\section{On-line system realisation}

Core simulators are traditionally run as batch programs with a rather cumbersome data input preparation procedure, especially for long predictive runs. This makes it difficult for operators in the control room to apply core simulators, and it was soon realised in the SCORPIO project that a simpler and more effective way of running predictive calculations was absolutely necessary.

The way of implementing the code and running it together with the whole SCORPIO system influences the total performance. For instance, the simulator runs on the fast minicomputer ND-500 while simulator output data are reorganised and stored into the data base administrated by the ND-100 minicomputer. The limit checking procedure is also performed here as well as the display of results after each time step in the calculation. All these series of tasks contribute to the total computing time. However, in SCORPIO parallel processing techniques are being used and new calculations are initiated on the ND-500 while the ND-100 completes the data storing, limit checking and picture display for the previous time step in a predictive calculation. Time delay due to data transfer is reduced through utilising shared memory and double buffering techniques.

\section{Future extensions}

\subsection{Core follow module}

The core follow module is an important part of a complete system. It will enable a better estimation of the actual core state by combining calculations and measurements. It will also update the distributions of xenon and iodine, so that the predictive calculations can always start from a correct initial condition. This module is expected to be ready for plant installation in the middle of 1987.

\subsection{Pin power calculation}

The coarse mesh simulator cannot calculate the detailed pin power distribution, but methods to obtain this from the coarse mesh results have been developed (Havranek et al. 1981). Until recently these methods have been too resource demanding for practical application in an on-line core surveillance system, but the rapid development in method simplification (Havranek 1982) and computer capacity has made the inclusion of such codes in the system a realistic possibility. 
Detailed pin power evaluation will enable a more accurate evaluation of the margins to the limits for the form factors $F_{Q}$ and $F_{\Delta H}$, which in turn may lead to a more flexible operation of the reactor. It may also be coupled with a fuel failure code and thus reduce the risk of fuel damage. Implementation of pin power calculation in SCORPIO will be made in cooperation with the University of Stuttgart.

\section{Conclusion}

A computerised surveillance system like SCORPIO has the potential of substantially increasing the quality and quantity of the information on core status and dynamic behaviour available to the operating staff. The benefits from this improved information are twofold. First of all, the safety of the plant is improved as undesired core conditions can be prevented more easily. Second, greater flexibility and efficiency in the operation of the plant is made possible. The presence of efficient computer hardware is a prerequisite when realising a complex system like SCORPIO. With today's minicomputers even demanding tasks like 3-dimensional core calculations can be performed. During the design and realisation of SCORPIO, the creation of a well balanced system compromising between computing speed and the complexity of the physics models included has been kept in mind. High performance of the total system as such is of special importance when serving as an operator aid in the control room. An effective and user-friendly input dialogue has been made to facilitate preparation of predictive simulation. The strategy generator has proved most useful for proposing good control strategies with the well defined objective of keeping a constant power distribution during a transient. Efficient implementation of the CYGNUS core simulator makes it possible to calculate a realistic transient of $20 \mathrm{~h}$ ahead in time within $4 \mathrm{~min}$ for a quarter core, 1120 nodes, PWR. Several trials can then be done within acceptable time limits before choosing a control strategy. The combination of an overview picture and detailed limit check and power distribution pictures is useful for identifying the critical parts of a transient.

SCORPIO's predictive mode of operation is fully developed, and has been transferred to the Ringhals Nuclear Power Plant units 2 (1984), 3 and 4 (1985). The main use of the system at Ringhals is operation planning, but it has also been found to be an efficient tool for increasing the operators' understanding of the dynamic behaviour of the reactor core in transient situations.

In cooperation with Duke Power Company (USA) and Norsk Data a predictive system for the reactor Catawba 2 (3411 MW thermal) will be installed at Duke's headquarters in the fall of 1987. During a six month period the system will be used by Duke's personnel, with the objective of evaluating the system's capability for enhancing the operation planning and surveillance of this reactor type.

The development of the core follow part of the system is nearly completed, and the coupling to the reactor instrumentation at unit 2 at Ringhals will take place in the middle of 1987.

\section{REFERENCES}

AHLIN, A., and EDEnius, M. (1977) CASMO-A fast transport assembly depletion code for LWR analysis. Trans. Am. Nucl. Soc., 26, 604.

HaVRANeK, M., and ElzmanN, J. (1981) A posteriori evaluation of the coarse-mesh calculation results in boiling water reactor dynamics. Nucl. Technology, 55, 568.

HavraneK, M. (1982) Das Konzept der schnellen Lupe. Private communication. 
TsuIKI, M., and AOKI, K. (1974) Modified one-group diffusion scheme for calculating gross power distribution in a BWR core. Journal of Nucl. Sci. and Technology, 11, 369.

Tsuiki, M., AOKI, K., YoshimURA, S. (1976) A new brief diffusion scheme for threedimensional calculation of a BWR core. Journal of Nucl. Sci. and Technology, 13, 541.

WeISs, Z. (1972) Nodal equations derived from invariant imbedding theory. Nuclear Science and Engineering, 48, 235. 